\title{
The Yeast Two-hybrid System: Criteria for Detecting Physiologically Significant Protein-Protein Interactions
}

\author{
Erica A. Golemis, Ilya Serebriiskii, and Susan F. Law \\ Division of Basic Research, Fox Chase Cancer Center, 7701 Burholme Ave \\ Philadelphia, PA 19111, USA
}

\begin{abstract}
In vivo transcription-based assays for protein-protein interactions such as the two-hybrid system are powerful methods for identifying novel proteins based on their physical association with known proteins of biological interest, or for characterizing the degree and nature of interactions between sets of proteins. Because of the complexity inherent in assays taking place within a living organism, a key issue for the effective use of two-hybrid approaches is the ability to determine whether apparent interactions are likely to be physiologically relevant.

In this article, a number of the different two-hybrid systems currently available for use will be reviewed. Then, taking as a model one such system, the Interaction Trap, examples of different reagents for use in varying the affinity range of detectable interactions will be outlined. Also set forth are a number of protocols to establish an appropriate set of conditions for either screening a library or analysing the interaction phenotype between protein sets. Finally, a number of general guidelines are suggested for trouble-shooting two-hybrid results, and for eliminating falsely positive interactions.
\end{abstract}

\section{Introduction}

In the last five years, two-hybrid systems have emerged as a ubiquitous tool in evaluation of the interaction between defined proteins, and as a screening method to identify novel partners for proteins of biological interest. One reason for this ascendence has proven to be the surprising utility of two-hybrid approaches for analysis of many classes of cellular proteins. A second reason is the relative convenience and speed of two-hybrid system based screens compared to biochemical methods for identifying protein partners. In contrast to other methods, no protein purification steps or development of antibodies are involved, the required cloning manipulations are minimal, and the interacting partner is obtained as a partial or complete cDNA. As a result of these advantages, a number of different two-hybrid systems have been developed, which, although they are all based on a similar theoretical paradigm, utilize somewhat differing components and screening strategies. An ever-increasing number of libraries are being generated with cDNAs derived from many different species and tissues, providing great power and flexibility to these two-hybrid systems. The goal of this article is to provide a general introduction to key elements common to all such two-hybrid systems, and then to focus on one variant, the Interaction Trap, discussing in some detail the steps one would take to establish optimal conditions to use this system with a protein of interest. 


\section{Paradigm}

Experiments performed by a number of groups through the 1980s led to the realization that many proteins were comprised of multiple sub-domains required for specific functions such as DNA binding, dimerization, transcriptional activation, and other properties. Based on this realization, in 1985, Brent and Ptashne demonstrated that it was possible to take individual domains with particular functions, such as the DNA-binding domain (DBD) from protein 1, and the transcriptional activation domain function from protein 2, and splice the two together. The novel chimaeric protein thus exhibits a DNA-binding specificity similar to protein 1 , and a protein 2-derived potential for activating transcription (1)(Figure 1A). In related work, studies on mechanisms of transcriptional control led to the finding that in some cases, proteins such as GAL80 which functioned as transcriptional regulators did not directly contact DNA or possess a covalently linked DNA-binding-domain. Instead, these regulatory proteins were brought to promoter sites via interaction with non-activating partner proteins such as GAL4 that possessed a DNA-binding-domain $(2,3)$. Exploiting these findings in 1988, Ma and Ptashne generated a synthetic transcriptional activator, which fused a heterologous activation domain to GAL80, allowing both interaction with GAL4 and activation of a GAL4-dependent transcriptional reporter (Figure 1B)(4).

Merging elements of these findings in 1989, Fields and Song described a scheme that established the functional paradigm for the two-hybrid system (5). In this scheme (Figure 1C), a first protein of interest, " $\mathrm{X}$ ", is synthesized as a fusion to a DNAbinding domain [ DBD-X], while a second protein, "Y", is synthesized as a fusion to an activation domain [AD-Y]. DBD-X and AD-Y are coexpressed in a host organism such as yeast which contains reporter genes under the transcriptional control of consensus binding sites for the DBD. If $\mathrm{X}$ and $\mathrm{Y}$ physically interact, this interaction brings the activation domain to the promoter of the reporter gene, activating its transcription. Although some other variables apply, in general, the strength of the activation provides an indirect measure of the strength of the interaction. This strategy has been widely applied to assess interactions between predefined X and Y protein sets, and has become a standard and powerful mechanism for identification of novel protein partners which interact with $X$ proteins of interest via the use of activation domain-fused cDNA libraries as the AD-Y element.

\section{Variants}

Since the original proposal of a two-hybrid strategy to analyse protein-protein interactions, a number of groups have developed reagent sets suitable for performing such studies (Table 1). The organism of choice as a host for two-hybrid applications has been Saccharomyces cerevisiae $(6,7,8,9)$. Although some systems have been developed on similar principles for use in mammalian cells with targeted protein pairs $(10,11)$, these have not attained broad use for such purposes as library screening, and will not be discussed here.

In the yeast systems, the DNA binding domain (DBD) function has standardly been supplied by the bacterial repressor protein LexA $(12,8,9)$, or by the yeast protein GAL4 $(13,6,7)$, although some other variants have been tried $(14,15)$. Activation domains (ADs) utilized have included those of GAL4 (6); an "acid blob", B42 $(16,8)$; and the viral protein VP16 $(17,9)$. To improve the accuracy and ease of library screening, two hybrid systems utilize two independent $D B D$-dependent reporter constructs in parallel. As primary reporters, two-hybrid systems utilize genes required for specific amino acid synthetic pathways, rendering growth on particular selective medium dependent on activation of the gene, thereby providing a very simple initial screening mechanism for positive interactors. The two genes of choice for this purpose have been $L E U 2$ required for growth on medium lacking leucine (8), and $H I S 3$, required for growth on medium lacking histidine $(6,7,9)$. All current systems include $l a c Z$ as a secondary reporter (18), allowing rapid blue-white 

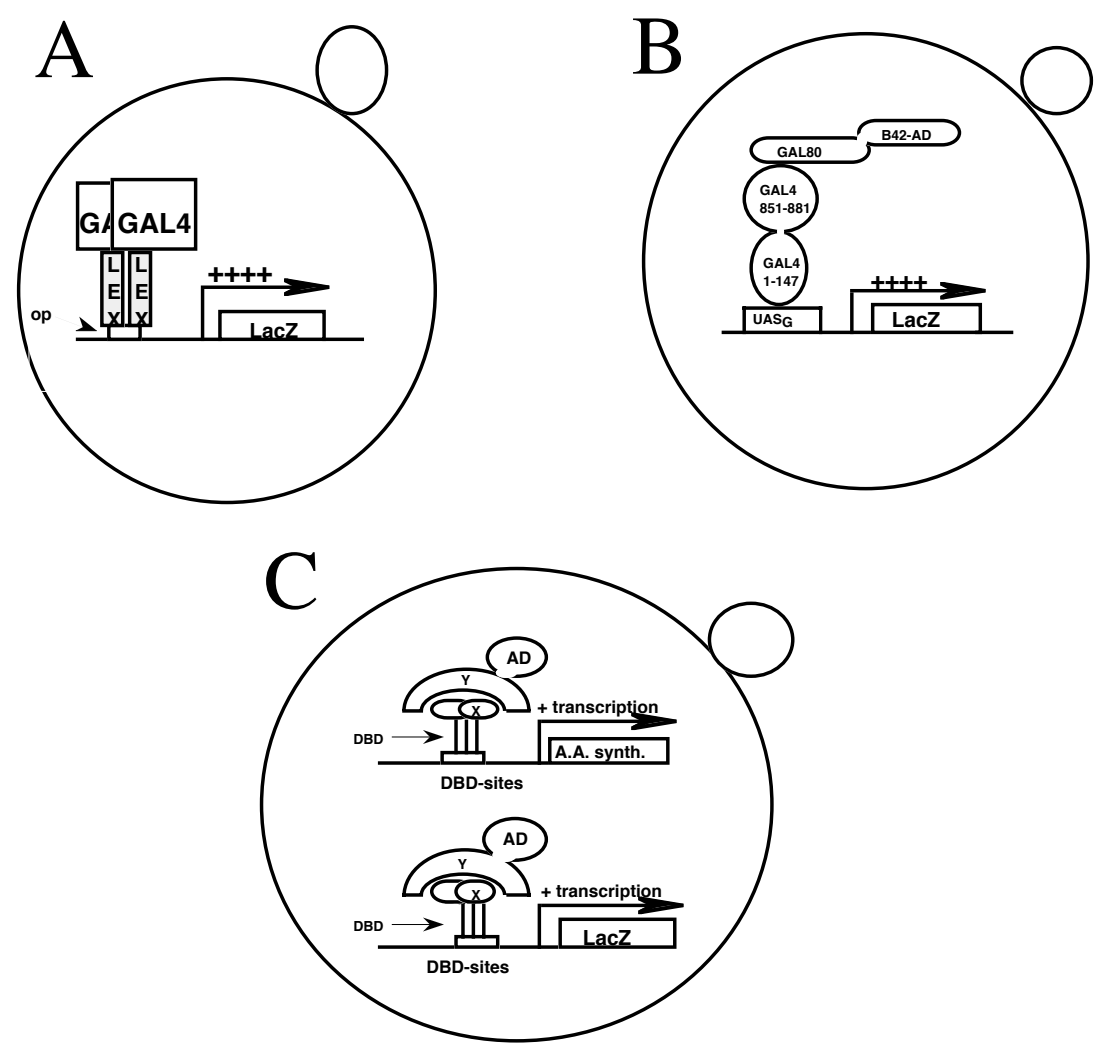

Figure 1. Development of the two-hybrid approach. All events are portrayed as taking place inside the host organism $S$. cerevisiae, budding yeast. A) Construction of a synthetic transcriptional activator with a defined DNA-binding specificity by fusion of the DNA-binding-domain from LexA with the activation domain from GAL4 (1). B) Construction of a synthetic transcriptional activator by fusion of the activation domain B42 (16) to the transcriptional repressor GAL80; this chimera interacts with the C terminal 80 amino acids of GAL4 (851-881) and activates transcription through a GAL4 binding site (UAS ${ }_{\mathrm{G}}$ ) (4). C) Paradigm for two-hybrid system, in which interaction between activation-domain-fused protein Y and DNA-binding-domain-fused protein $\mathrm{X}$ causes activation of a reporter gene under transcriptional control of binding sites for the DNA-binding-domain (5).

confirmation of positives on plates containing 5-bromo-4-chloro-3-indolyl- $\beta$-Dgalactoside (X-Gal) as well as approximate quantitation of interaction affinity using a $\beta$-galactosidase assay. Detailed protocols have been published to provide step-bystep instructions for performance of two-hybrid library screens, for both GAL4 (19) and and LexA (20) based screening systems.

\section{Interactions Detected by Two-Hybrid Systems}

While no extensive database of biologically meaningful interactors isolated by twohybrid screens as yet exists, a survey of some of the published literature is instructive as to what classes of protein-protein interactions have been successfully studied by two-hybrid screening mechanisms. A number of these studies are summarized in Table 2, where the interacting proteins are grouped based on the nature of the DBDfused bait protein used. Certain protein classes have been used extensively in twohybrid screens and have yielded information about interactors that appears to be 
Table 1. List of two-hybrid system variants. A number of groups have developed different sets of reagents based on the two-hybrid paradigm. Literature references are described in the text.

\begin{tabular}{|c|c|c|c|c|c|}
\hline $\begin{array}{l}\text { Lab. of } \\
\text { Origin }\end{array}$ & System & Host & $\begin{array}{l}\text { DNA Binding } \\
\text { Domain }\end{array}$ & $\begin{array}{l}\text { Activation } \\
\text { Domain }\end{array}$ & Selection \\
\hline S.Fields & $\begin{array}{l}\text { Two-Hybrid } \\
\text { Matchmaker }\end{array}$ & Yeast & GAL4 & GAL4 & Activation of lacZ, HIS3 \\
\hline R.Brent & $\begin{array}{l}\text { Interaction } \\
\text { Trap }\end{array}$ & Yeast & LexA & $\begin{array}{l}\text { "Acid Blob" } \\
\text { B42 }\end{array}$ & Activation of $L E U 2, \operatorname{lac} Z$ \\
\hline S.Elledge & $\begin{array}{l}\text { "Improved } \\
\text { Two-Hybrid" }\end{array}$ & Yeast & GAL4 & GAL4 & Activation of $H I S 3$, lacZ \\
\hline S.Hollenberg & $\begin{array}{l}\text { "Modified } \\
\text { Two-Hybrid" }\end{array}$ & Yeast & LexA & VP16 & Activation of HIS3, lacZ \\
\hline $\begin{array}{l}\text { E.Fearson/ } \\
\text { C.Dang }\end{array}$ & KISS & $\begin{array}{l}\text { Mammalian } \\
\text { Cells }\end{array}$ & GAL4 & VP16 & Activation of $C A T, h y g \mathrm{R}$ \\
\hline S.Weissman & $\begin{array}{l}\text { Contingent } \\
\text { Replication }\end{array}$ & $\begin{array}{l}\text { Mammalian } \\
\text { Cells }\end{array}$ & GAL4 & VP16 & $\begin{array}{l}\text { Activation of T-Ag, } \\
\text { Replication of plasmids }\end{array}$ \\
\hline
\end{tabular}

physiologically significant. Many cell cycle regulatory proteins have been utilized, including cyclin dependent kinases, cyclins, and negative regulatory factors. Small GTPases and other cytoplasmic or loosely membrane associated signaling molecules have also been used to identify their interacting regulatory molecules. Aditionally, serine threonine-targeted protein kinases, and in some cases tyrosine-targeted protein kinases have been used (although in some cases the latter are known to be partially toxic to yeast). In other studies, DNA repair molecules such as $R A D$ genes, splicing factors, and a large number of genes associated with transcriptional activation, transcriptional silencing, and transcriptional repression have been productively employed to identify relevant partner proteins. Finally, a diverse group of other proteins have been examined using two-hybrid technology, including the tumor suppressor $\mathrm{Rb}$, DNA recombination proteins, viral proteins, apoptotic regulators and others.

What affinity range of interactions are detected by two-hybrid approaches? In recent work by Estojak et al., protein sets for which the affinity of association had previously been biochemically determined were fused in all possible combinations to LexA or to an activation domain. These were used with a series of LexA-operator$L a c Z$ or $L e x A$-operator-LEU2 reporters to establish the degree to which apparent affinity of protein-protein interaction determined by the two-hybrid approach paralleled association affinity determined in vitro (21). Results of this study suggested that interactions thought to occur with an affinity of less than 1-5 $\mu \mathrm{M}$ (approx.), which are believed to be non-physiological, were poorly or not at all detected in the Interaction Trap using any of the $L a c Z$ or $L E U 2$ reporters. In contrast, interactions that occur with affinities of lower than $20 \mathrm{nM}$, such as the heterodimerization between the oncoprotein Myc and its partner Max, or the homodimerization between lambda cI repressor molecules, were detected strongly. Additionally, protein affinities ranging from $1 \mathrm{nM}$ to $1 \mathrm{uM}$ could generally be discriminated, with rank order similar to that determined in vitro. However, in quantitation of affinity, increase in B-galactosidase activity (reflecting activation of the lacZ gene) was not precisely colinear with fold increase in affinity, suggesting that caution should be used in extrapolating exact affinities from two-hybrid phenotype. Finally, different reporter constructs apparently had different thresholds of affinity for detecting interactions. Those with transcription dependent on fewer LexA-operators (such as EGY191 yeast, or pRB1840) failed to identify as positive some of the lowest affinity interactions. However, these less sensitive reporters had the advantage in some cases of suppressing "background" transcriptional activation by the DBD-fused component, making it sometimes possible to discriminate interactions that would be obscured using more sensitive reporters. Cumulatively, these results together with the record of known two-hybrid 
applications (Table 2) indicate that two-hybrid approaches can be used flexibly with many classes of biologically interesting proteins.

\section{Issues in Planning Two-Hybrid Screens}

Although many proteins have been studied successfully with two-hybrid technology, not all proteins are appropriate for use in a two-hybrid system. Because of the significant effort required in performing and analyzing the results of a two-hybrid screen, before initiating such a screen with a protein of interest (termed a "bait"), it is worth carefully considering some properties of the bait that may specifically indicate that a given protein is not likely to be used productively. The following describes problems that have occurred with some proteins used in two-hybrid systems, and represent the types of issues an investigator should consider before launching such a screen.

As currently construed, two-hybrid systems depend on selective transcriptional activation of a reporter mediated by interaction of a bait with a library encoded, AD-fused partner protein. For this approach to work, the potential bait must be capable of localizing to the cell nucleus. It must not possess intrinsic ability to strongly activate transcription of the reporter constructs. It must also be able to bind to DBD consensus sites upstream of the reporter constructs. For these reasons, proteins known to be integral membrane components or otherwise known to localize to plasma or organellar membranes may cause difficulties if fused to DBD domains in their native states, as a large proportion of these proteins may be sequestered from the binding sites. Transmembrane and membrane targetting sequences should generally be truncated.

Proteins that are strong transcriptional activators are also unlikely to be successfully employed, because reporter transcripts will be abundantly produced in the absence of a library component. It should be noted that some proteins that normally do not function as transcriptional activators (for example, some cytoplasmic protein kinases) will, when fused to a DBD and expressed in yeast, in fact activate transcription quite strongly. Hence, any protein which is to be used as a bait should first be tested for spurious activation of two-hybrid reporters.

If a potential bait possesses its own DBD (i.e. is normally a DNA-binding protein), it is possible that this DBD will contribute to sequestration of the bait to its preferred consensus sites, occurring at random in yeast DNA, thereby diminishing the quantity of DBD-bait capable of binding upstream of the reporter gene constructs. In some cases, removal of the native DNA-binding domain from the bait results in improved behavior in a two-hybrid system (see reference 22).

If the intended bait is a higher-eukaryotic protein which is normally posttranslationally processed, and this processing is thought to be important for interaction with partner proteins, a major concern would be that the appropriate modification may not occur in yeast. Examples of such modification might include glycosylation, or phosphorylation by a specific serine/threonine or tyrosine kinase. "Tri-hybrid" systems, in which an additional kinase is co-expressed with the bait to provide the appropriate modification have been developed to help circumvent some of these problems (23). While some claims of successful two-hybrid use have been made for proteins normally expressed extracellularly (24), it is not clear how common such success is likely to be.

While most proteins can be stably expressed as fusion proteins to a LexA or GAL4 DBD in yeast, some proteins are either poorly expressed, or are clipped by yeast proteases so that the predominant population of $\mathrm{DBD}$-fusion in a cell is significantly less than full length. These problems are more common for large fusion domains (i.e. $>50 \mathrm{kD}$ ). Prior to commencing a screen, it is thus generally advisable to use antibody to the DBD in a western blotting analysis of yeast lysates containing fusion proteins to ensure that detectable quantities of an appropriately sized bait are produced. 
Table 2. List of protein-protein interactions identified or analyzed by two-hybrid techniques.

\begin{tabular}{|c|c|c|c|c|}
\hline Bait Type & DBD Fused Protein & AD Fused Protein & Prey Type & Lib./ set \\
\hline a & Grb2 & $\operatorname{sos}$ & gef & $\mathrm{s}$ \\
\hline ag & CREB/ATF & Ig var.domain & $\mathrm{ab}$ & s \\
\hline apopt & $\mathrm{Bcl}-2$ & R-Ras & $\mathrm{g}$ & 1 \\
\hline ca & Calmodulin & Spc110 & ${ }_{0}^{\circ}$ & $\mathrm{s}, 1$ \\
\hline ca & Calmodulin & Spc110p & o & 1 \\
\hline cdc & $\mathrm{Cdc} 23 \mathrm{p}$ & Cdc $27 \mathrm{p}$ & cdc & $\mathrm{s}$ \\
\hline $\mathrm{cdk}$ & $\mathrm{Cdk} 2$ & Rbr-2 & $\mathrm{Rb}$ & 1 \\
\hline $\mathrm{cdk}$ & CDK4 & p16ink4 & 0 & 1 \\
\hline $\mathrm{cdk}$ & Cdk6 & p16 & o & 1 \\
\hline cdk & pho2 & pho4 & tr & s \\
\hline cdk & $\mathrm{HsCdc} 2, \mathrm{HsCdk} 2$ & $\mathrm{Cdi} 2$ & 0 & s \\
\hline cdk & DmCdc2, ScCdc28 & $\mathrm{Cdi} 2$ & o & $\begin{array}{l}\mathrm{s} \\
\mathrm{s}\end{array}$ \\
\hline $\mathrm{cdk}$ & DmCdc2c, HsCdk3 & Cdi 2,4 & o & $\mathrm{s}$ \\
\hline $\mathrm{cdk}$ & DmCdc2c, ScCdc28 & $\mathrm{Cdi} 3,5$ & cyclin & s \\
\hline $\mathrm{cdk}$ & $\mathrm{DmCdc} 2$ & Cdi3, 7 & cyclin & s \\
\hline cdk & $\mathrm{HsCdk} 3,4$ & Cdi3 & cyclin & $\mathrm{s}$ \\
\hline cyclin & cyclindi & p27 & o & $\mathrm{s}$ \\
\hline cyclin & Cdi5 & $\mathrm{Cdi} 2$ & o & $\mathrm{s}$ \\
\hline cyp & cycA & beta-tubulin & o & 1 \\
\hline cyp & cycB & CAML & ca & 1 \\
\hline dbp & CENP-B & CENP-B & dbp & s \\
\hline $\mathrm{dbp}$ & $\operatorname{Max}$ & Myc & $\begin{array}{l}\text { aop } \\
\text { o }\end{array}$ & $\begin{array}{l}\mathrm{s} \\
\mathrm{s}\end{array}$ \\
\hline $\mathrm{dbp}$ & RBP3 & prot $\mathrm{H}$ & dbp & 1 \\
\hline $\mathrm{g}$ & $\mathrm{R}$-ras & RalGDS & gds & 1 \\
\hline ğ & Rab3A & Rabin & o & 1 \\
\hline 莺 & Ras & RalGDS & $\mathrm{gd}$ & 1 \\
\hline g & $\begin{array}{l}\text { ras } \\
\text { ras }\end{array}$ & $\begin{array}{l}\text { cdc } 25 \\
\text { con }\end{array}$ & gef & $\begin{array}{l}1 \\
\text { s }\end{array}$ \\
\hline $\mathrm{g}$ & Ras & Raf & $\mathrm{pk}$ & s \\
\hline $\mathrm{g}$ & ras p21 & RGL & gds & 1 \\
\hline g & ras1, ras2 & byr2 & $\mathrm{pk}$ & $1, \mathrm{~s}$ \\
\hline g & ras1 1 ias & scd1 1 & o & $\begin{array}{l}1, \mathrm{~s} \\
\mathrm{l}\end{array}$ \\
\hline g & Ras2 & $\mathrm{CDC} 25$ & cdc & s \\
\hline $\mathrm{g}_{\mathrm{g}}$ & H-,K-ras, Rap1 & RalGDS & gds & s \\
\hline g & cdc $42 \mathrm{sp}$ & scd1, scd2 & 0 & $\mathrm{~s}$ \\
\hline g-p & Ste4 & Ste 18 & g-p & $\mathrm{s}$ \\
\hline $\mathrm{pk}$ & $\begin{array}{l}\text { byr } 2 \\
\text { ben }\end{array}$ & H-ras & ${ }_{\mathrm{g}}^{\mathrm{g}-\mathrm{p}}$ & $\mathrm{s}$ \\
\hline pk & $\begin{array}{l}\text { byr1 } \\
\text { byr1 }\end{array}$ & $\begin{array}{l}\text { spk1 } \\
\text { spks }\end{array}$ & o & s \\
\hline $\mathrm{pk}$ & Bruton's ptk & Fyn & $\mathrm{pk}$ & 1 \\
\hline pk & cdc7 7 & DBF4 & cyclin & $\mathrm{s}$ \\
\hline $\mathrm{pk}$ & Fus & Ste5 & st & $\mathrm{s}$ \\
\hline $\mathrm{pk}$ & MO15 & cyclinH & cyclin & 1 \\
\hline $\mathrm{pk}$ & p52shc & PTP-PEST & $\mathrm{pp}$ & $\mathrm{s}$ \\
\hline $\mathrm{pk}$ & p110 of PI 3-kinase & p85 of PI 3-kinase & pk & s \\
\hline pk & $\begin{array}{l}\text { PKC } \\
\text { PKC } 1 \text {-KIndase }\end{array}$ & pICK1 1 - Jinase & $\begin{array}{l}\mathrm{pk} \\
\mathrm{o}\end{array}$ & 1 \\
\hline $\mathrm{pk}$ & Raf & Ras & $\mathrm{g}$ & $\mathrm{s}$ \\
\hline $\mathrm{pk}$ & Raf & Rap1 & $\mathrm{g}$ & $\mathrm{s}$ \\
\hline $\begin{array}{l}\mathrm{ph} \\
\mathrm{pk}\end{array}$ & Raf & MEK & pk & s \\
\hline $\mathrm{pk}$ & MEK & Raf & $\mathrm{pk}$ & $\mathrm{s}$ \\
\hline pk & Raf-1 & HS1 & $14-3-3$ & i \\
\hline $\mathrm{pk}$ & Raf-1 & PLA2 & $14-3-3$ & 1 \\
\hline $\mathrm{pk}$ & Raf-1 & $14-3-3$ beta isoform & $14-3-3$ & 1 \\
\hline $\mathrm{pk}$ & SNF1 & MSN3 (STD1) & $\begin{array}{l}14-5-3 \\
0\end{array}$ & 1 \\
\hline $\begin{array}{l}\mathrm{pk} \\
\mathrm{pk}\end{array}$ & SNF1 & SIP1, SIP2, SIP3, SIP4 & $\begin{array}{l}0 \\
0\end{array}$ & 1 \\
\hline $\mathrm{pk}$ & c-abl & Crk & $\begin{array}{l}0 \\
\mathrm{a}\end{array}$ & 1 \\
\hline $\mathrm{pk}$ & Fyn & p62 & $\begin{array}{l}\mathrm{a} \\
\mathrm{a}\end{array}$ & 1 \\
\hline $\mathrm{pk}$ & ANP & novel & $\begin{array}{ll}\mathrm{a} \\
\mathrm{pk}\end{array}$ & 1 \\
\hline $\mathrm{pp}$ & Cap1 delta & Cap1 AID & pp & $\mathrm{s}$ \\
\hline $\mathrm{pp}$ & Capl delta & Cmd1 & $\mathrm{Ca}$ & $\mathrm{s}$ \\
\hline $\mathrm{Rb}$ & p10RB & PP-1 alpha 2 & $\mathrm{pp}$ & 1 \\
\hline $\mathrm{Rb}$ & p110RB & T-antigen SV-40 & $\mathrm{pp}$ & 1 \\
\hline $\mathrm{Rb}$ & p110RB & p110RB & $\mathrm{Rb}$ & $\begin{array}{l}1 \\
\mathrm{~s}\end{array}$ \\
\hline $\mathrm{Rb}$ & p110RB & p84 & $\mathrm{o}$ & 1 \\
\hline $\mathrm{Rb}$ & p110RB & CycD1 & cyclin & $\mathrm{s}$ \\
\hline $\mathrm{Rb}$ & p110RB & BRG1 & tr & $1, \mathrm{~s}$ \\
\hline 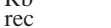 & RAG1 & SRP1 & o & l, s \\
\hline recep & ins. rec & IRS-1 & o & $\mathrm{s}$ \\
\hline recep & Insulin rec. & LIM cont. prot & o & 1 \\
\hline recep & mPPAR & $\mathrm{hRXR}$ & recep & $\mathrm{s}$ \\
\hline $\begin{array}{l}\text { recep } \\
\text { recep }\end{array}$ & TGF-beta R-I & TGF-beta R-II & recep & s \\
\hline recep & TNFR2 & TRAF1, TRAF2 & o & 1 \\
\hline recep & TNFR-1 & TNFR-1 & recep & $\mathrm{s}, 1$ \\
\hline recep & TNFR-1IC & TRAP-1 1 & hsp & 1 \\
\hline rep & Rad51 & $\operatorname{Rad} 51$ & rep & $\mathrm{s}$ \\
\hline rep & Rad51 & Rad52 & rep & s \\
\hline rep & $\operatorname{Rad} 7$ & SIR3 & $\mathrm{dbp}$ & s \\
\hline spl & HRH1 & SR protein family & $\mathrm{dbp}$ & s \\
\hline spl & SC 35 & SC $35, \mathrm{U} 2 \mathrm{AF} 35, \mathrm{U} 1-70 \mathrm{~K}$ & spl & $1, \mathrm{~s}$ \\
\hline spl & SC 35 & SF2/ÄSE Tra Tra-2 & $\mathrm{spl}$ & $\mathrm{s}$ \\
\hline spl & SF2/ASF & SC 35, SF2/ASF & spl & s \\
\hline spl & SF2/ASF & U2AF35, U1-70K & spl & $\begin{array}{l}\mathrm{s} \\
\mathrm{s}\end{array}$ \\
\hline spl & $\begin{array}{l}\text { SPP91 } \\
\text { SP }\end{array}$ & PRP11 & $\mathrm{spl}$ & $\mathrm{s}$ \\
\hline
\end{tabular}




\begin{tabular}{|c|c|c|c|c|}
\hline Bait Type & DBD Fused Protein & AD Fused Protein & Prey Type & Lib./ set \\
\hline $\mathrm{spl}$ & $\mathrm{U} 2 \mathrm{AF} 35$ & SC35, SF2/ASF, Tra & spl & $\mathrm{s}$ \\
\hline $\begin{array}{l}\text { spl } \\
\text { st }\end{array}$ & $\mathrm{U} 2 \mathrm{AF} 35$ & U2AF35, U2AF65, Tra-2 & spl & s \\
\hline $\begin{array}{l}\text { st } \\
\text { st }\end{array}$ & $\begin{array}{l}\text { Vav } 11 \\
\text { STE11 }\end{array}$ & $\begin{array}{l}\text { MnRNAP K } \\
\text { STE5, STE7, FUS3 } 3\end{array}$ & $\begin{array}{l}\mathrm{o} \\
\text { st }\end{array}$ & $\begin{array}{l}1 \\
\mathrm{~s}\end{array}$ \\
\hline st & STE5 & STE7, STE1 1 , FUS3 3 & st & $\mathrm{s}$ \\
\hline st & STE7 & STE5, STE7, STE11, FUS3 & st & $\mathrm{s}$ \\
\hline $\begin{array}{l}\text { st } \\
\text { t.ad }\end{array}$ & $\begin{array}{l}\text { FUS3 } \\
\text { GCN5 }\end{array}$ & $\begin{array}{l}\text { STE7 } \\
\text { ADA2 }\end{array}$ & st & $\mathrm{s}$ \\
\hline tr & $\mathrm{B}$ (maize) & ADA2 & t.ad & $\mathrm{s}$ \\
\hline $\operatorname{tr}$ & Dr1 & TBP & tr.f & s \\
\hline $\operatorname{tr}_{\text {tr }}$ & Not1 & Not2, Not4 & tr & $\mathrm{s}$ \\
\hline $\operatorname{tr}_{\text {tr }}$ & Not3 & Not2, Not4 & tr & $\mathrm{s}$ \\
\hline $\begin{array}{l}\text { tr } \\
\text { tr }\end{array}$ & $\begin{array}{l}\text { Sho80 } \\
\text { SIR4 }\end{array}$ & $\begin{array}{l}\text { Pho4 } \\
\text { SIR4 }\end{array}$ & $\begin{array}{l}\operatorname{tr} \\
\operatorname{tr}\end{array}$ & $\begin{array}{l}\mathrm{s} \\
\mathrm{s}\end{array}$ \\
\hline & RAP1 & RIF & o & 1 \\
\hline tr.f. & Fos & Jun & tr.f & $\mathrm{s}$ \\
\hline $\begin{array}{l}\text { tr.f. } \\
\text { tr.f }\end{array}$ & $\begin{array}{l}\text { RBTN2 } \\
\text { TAL1, TAL2 }\end{array}$ & $\begin{array}{l}\text { TAL1 } \\
\text { RBTN1.RBTN2 }\end{array}$ & tr.f & $\mathrm{s}$ \\
\hline tr.f & $\begin{array}{l}\text { 1AL1, IAL2 } \\
\text { LYL1 }\end{array}$ & $\begin{array}{l}\text { RBIN1, RBIN2 } \\
\text { RBTN1, RBTN2 }\end{array}$ & $\begin{array}{l}\text { tr.t } \\
\text { tr.f }\end{array}$ & $\begin{array}{l}\mathrm{s} \\
\mathrm{s}\end{array}$ \\
\hline $\begin{array}{l}\text { tr.f. } \\
\text { trf }\end{array}$ & E47 & RBTN1, TAL1 & tr.f & $\mathrm{s}$ \\
\hline tr.f. & TAL1 & E47 & tr.f & $\mathrm{s}$ \\
\hline $\begin{array}{l}\text { tr.f. } \\
\text { tr.f }\end{array}$ & E12 & Id-2 & tr.f & $\mathrm{s}$ \\
\hline tr.f & $\begin{array}{l}\text { E12 } \\
\text { MyoD }\end{array}$ & MyoD & $\begin{array}{l}\operatorname{tr} . f \\
\text { trf }\end{array}$ & s \\
\hline tr.f & hTAF & $\begin{array}{l}\text { ET, E4/ } \\
\text { hTBP }\end{array}$ & tr.f & $\mathrm{s}$ \\
\hline tr.f & TFB1 & SSL1 & $\begin{array}{l}\text { tr. } \\
\text { rep }\end{array}$ & 1 \\
\hline & HSF2 & HSF2 & tr.f & $\mathrm{s}$ \\
\hline $\begin{array}{l}\text { transl.a } \\
\text { tta }\end{array}$ & $\begin{array}{l}\text { PET54, } \\
\text { protX }\end{array}$ & $\begin{array}{l}\text { PET122, } 494 \\
\text { XAP } 1\end{array}$ & transl.a & s \\
\hline $\begin{array}{l}\text { tatal } \\
\text { viral }\end{array}$ & $\begin{array}{l}\text { protX } \\
\text { Pr5sag }\end{array}$ & $\begin{array}{l}\text { XAP-1 } \\
\text { Pr55 gag }\end{array}$ & rep & \\
\hline viral & M-MuLV & M-MuLV & $\begin{array}{l}\text { Viral gag) } \\
\text { viral (gag) }\end{array}$ & $\begin{array}{l}\mathrm{s} \\
\mathrm{s}\end{array}$ \\
\hline viral & EBNA-2 & EBNA-2 & viral & $\mathrm{s}$ \\
\hline $\begin{array}{l}\text { viral } \\
\text { viral }\end{array}$ & $\begin{array}{l}\text { C (hepadnavirus) } \\
\text { E4 ORF6/7 }\end{array}$ & $\begin{array}{l}\text { C (hepadnavirus) } \\
\text { E2F-1 }\end{array}$ & viral & $\mathrm{s}$ \\
\hline $\begin{array}{l}\text { viral } \\
\text { viral }\end{array}$ & $\begin{array}{l}\text { E } \\
\text { Nef }\end{array}$ & $\begin{array}{l}\mathrm{E} 2 \mathrm{~F}-1 \\
\text { beta-COP }\end{array}$ & $\begin{array}{l}\text { tr.t. } \\
0\end{array}$ & $\begin{array}{l}\mathrm{s} \\
1\end{array}$ \\
\hline o & SNF4 & SNF1 & $\mathrm{pk}$ & $\mathrm{s}$ \\
\hline $\begin{array}{l}0 \\
0\end{array}$ & $\begin{array}{l}\text { cdil11 } \\
\text { scd2 }\end{array}$ & $\begin{array}{l}\text { Cdi3 } \\
\text { scd1 }\end{array}$ & $\begin{array}{l}\text { cyclin } \\
\mathrm{o}\end{array}$ & $\begin{array}{l}\mathrm{s} \\
\mathrm{s}\end{array}$ \\
\hline & & & & \\
\hline o & FKBP12-rapamycin & $\begin{array}{l}\text { RAPT1 } \\
\text { TOR2 }\end{array}$ & o & 1 \\
\hline $\begin{array}{l}0 \\
0\end{array}$ & $\begin{array}{l}\text { FKBP12-rapamycin } \\
\text { period }\end{array}$ & $\begin{array}{l}\text { 1OR2 } \\
\text { period }\end{array}$ & $\begin{array}{l}\mathrm{o} \\
\mathrm{o}\end{array}$ & $\begin{array}{l}\mathrm{s} \\
\mathrm{s}\end{array}$ \\
\hline o & TRAF1 & TRAF1, TRAF2 & o & s \\
\hline o & TRAF2 & TRAF1, TRAF2 & o & $\mathrm{s}$ \\
\hline o & BCY1 & BCY1 & 0 & $\mathrm{~s}$ \\
\hline o & p53 & p53 & o & 1 \\
\hline
\end{tabular}

Interacting pairs listed are taken from published articles (references available upon request), and were obtained using either GAL4 or LexA-based yeast two-hybrid systems. Abbreviations for classifications of interacting proteins are, $a$, adaptor protein; $a b$, antibody; $a g$, antigen; apopt, apoptosis-related protein; $c a$, calcium regulated protein; $c d c$, cell division control; $c d k$, cyclin dependent kinase; $c y p$, cyclophilin protein; $d b p$, DNA binding protein; $g$, GTPase; $g$ - $p$, G-protein; $g d s$, GDP-dissociation stimulator; gef, GTP-GDP exchange factor; $o$, other; $p k$, protein kinase; $p p$, protein phosphatase; $r e c$, recombination protein; recep, receptor protein; rep, DNA repair factor; $s p l$, splicing factor; st, signal transduction related; $t$. ad., transcription adaptor; $t r$, transcriptional regulator; $t r . f$., transcription factor; transl. $a$., translational activator; $t$ ta, transcription transactivator. Abbreviations for the methods used to detect the interaction are $s$, predefined Set of proteins; $l$, library screen.

A small number of higher eukaryotic proteins appear to be toxic to greater or lesser degrees when expressed in yeast. Such toxic properties can generally be detected while performing initial transformations to characterize DBD-baits, since plasmids expressing such proteins will yield significantly fewer colonies than control vector. Alternatively, yeast expressing the toxic DBD-fusion will generally grow at a much slower rate and transform more poorly than yeast expressing non-toxic proteins. While such toxicity can sometimes be exploited to useful purpose (25), it frequently results in screening difficulties, and steps should thus be taken to minimize toxic effects, as outlined below.

One possible variable of unknown importance is the interaction of heterologously expressed DBD-fused proteins with endogenous yeast proteins. Because of evolutionary conservation, an increasingly large number of mammalian proteins 


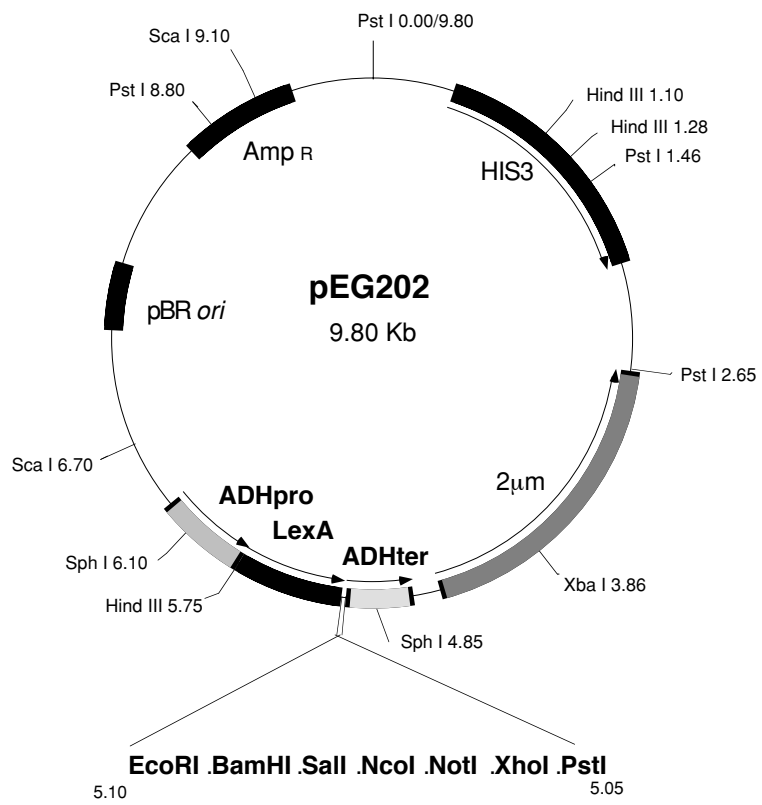

Figure 2. LexA-fusion vector. pEG202, a derivative of Lex202 + PL (16), uses the strong alcohol dehydrogenase promoter ( $A D H$ pro) to express bait proteins as fusions to the DNA-binding protein LexA. A number of restriction sites immediately upstream of ADHter are available for insertion of coding sequences. The plasmid contains the HIS 3 selectable marker and the $2 \mu \mathrm{m}$ origin of replication to allow propagation in yeast, and the ampicillin resistance gene $\left(\mathrm{amp}^{\mathrm{R}}\right)$ and the $\mathrm{pBR}$ origin (ori) of replication to allow propagation in Escherichia coli. Numbers indicate relative map positions.

have been shown to be capable of complementing mutations in their yeast homolog, and, by inference, to be capable of interacting with yeast partner proteins. Hence, a formal possibility is that some apparent protein-protein interactions may in fact reflect ternary or higher order complexes involving yeast components. Similarly, the detection of some interactions between mammalian proteins may be inhibited or blocked because one component of the complex is sequestered by interaction with an abundant yeast protein.

Finally, it is often desirable or necessary to attempt two-hybrid screens with truncated forms of proteins. Wherever possible, these truncations should be guided by preexisting knowledge of protein domain structure, since inappropriate truncations may lead to synthesis of partially or largely unfolded proteins that isolate false positive proteins such as Hsp70 from library screens.

\section{Establishing Two-Hybrid Assay Conditions for a Protein of Interest Using the Interaction Trap}

While proteins that strongly activate transcription are generally not appropriate for two hybrid screening, many proteins which are weak or moderate activators of transcription can be productively utilized, given some initial adjustment of screening parameters. This is most commonly accomplished by varying the sensitivity of the reporter genes utilized, although in some cases it can be addressed by altering the expression of the bait and library proteins. The exact sequence of events to establish screening conditions will vary from system to system. As an example of the general approach, we will here use the Interaction Trap as a model to demonstrate the steps 


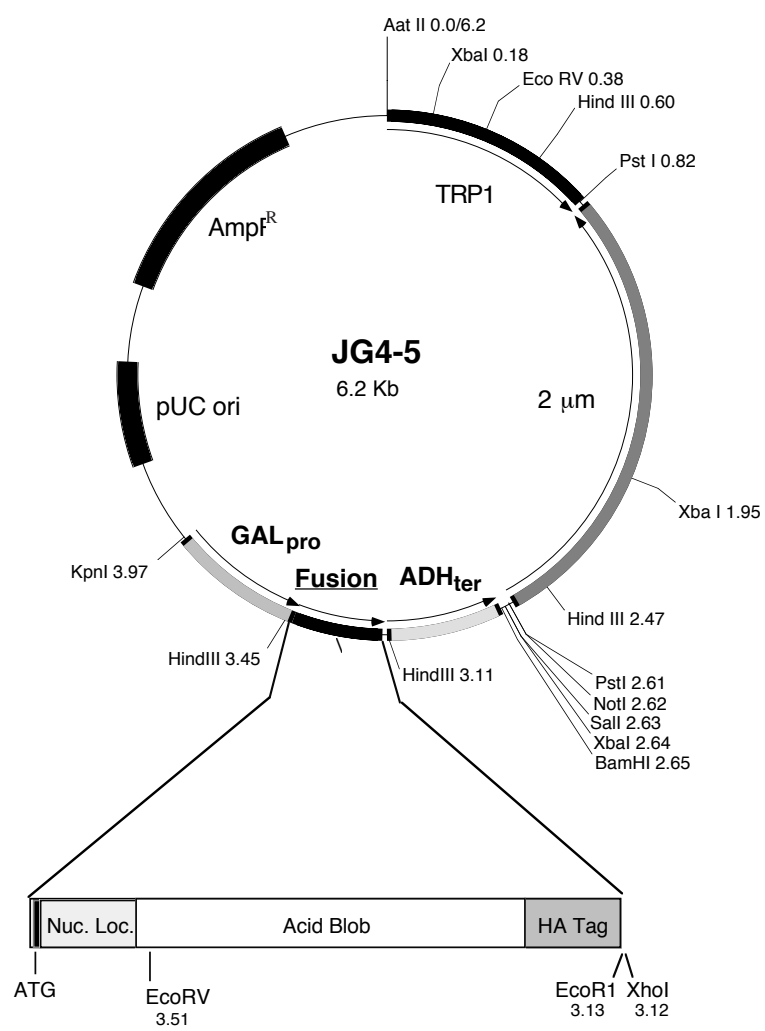

Figure 3. Activation domain-fusion vector. pJG4-5 (8) expresses cDNAs or other coding sequences inserted into the unique $E c o \mathrm{R} I$ and $X h o$ I sites as a translational fusion to a cassette consisting of the SV40 nuclear localization (nuc. loc.) sequence (PPKKKRKVA), the acid blob B42, and the hemagglutinin (HA) epitope tag (YPYDVPDYA). Expression of sequences is under the control of the $G A L l$ inducible promoter. The plasmid contains the TRP1 selectable marker and the $2 \mu \mathrm{m}$ origin to allow propagation in yeast, and the ampicillin resistance $\left(\mathrm{amp}^{\mathrm{R}}\right)$ gene and the $\mathrm{pUC}$ origin (ori) to allow propagation in E. coli. Numbers indicate relative map positions.

one would use to establish screening conditions for a novel DBD-bait protein. Detailed descriptions of how one would perform such a screen are presented in other forums, most notably Current Protocols in Molecular Biology (see reference 20).

\section{Reagents}

The Interaction Trap two-hybrid system was initially developed using a single core set of reagents. The plasmid pEG202 uses the strong alcohol dehydrogenase promoter to express baits as fusions to the DBD function of LexA (Figure 2). The plasmid pJG4-5 uses the galactose-inducible GAL1 promoter to express predefined interacting proteins or cDNA libraries as fusions to a cassette including a nuclear localization sequence, an activation domain, and a hemagglutinin epitope tag (Figure 3). The plasmid pSH18-34 contains 8 operator sites for LexA binding upstream of the lac Z reporter gene (Figure 4). Finally, in the yeast strain EGY48, homologous recombination has been used to replace upstream activating sequences for the LEU2 gene with 6 operators for LexA, rendering growth on medium without leucine 


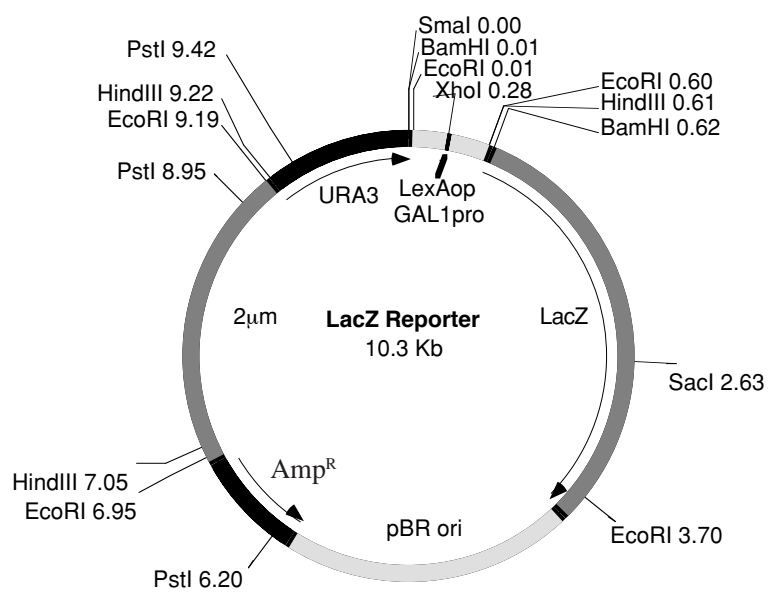

Figure 4. LacZ-Reporter. pRB1840, pJK103, and pSH18-34 are all derivatives of LR1del1 (28) containing 8,2 , or 1 operator for LexA binding inserted into a unique XhoI site located in the minimal GALI promoter ( 0.28 on map). The plasmid contains the URA3 selectable marker and the $2 \mu \mathrm{m}$ origin to allow propagation in yeast, and the ampicillin resistance $\left(\mathrm{amp}^{\mathrm{R}}\right)$ gene and the $\mathrm{pBR} 322$ origin (ori) to allow propagation in E. coli. Numbers indicate relative map positions.

dependent on activation through a LexA binding site. Screening for positive interactions is done by transforming a pJG4-5-based library into EGY48 yeast containing a pEG202 fusion and pSH18-34, and assaying for simultaneous activation of Leu2 and lacZ reporters in a galactose-dependent manner.

As large numbers of researchers began to utilize this two-hybrid system, individuals modified the original vector and strain components to increase the general flexibility of the Interaction Trap and render it usable with a greater range of proteins of interest. A number of these additional reagents are listed in Table 3. Reporter sensitivity levels were varied by the simple device of altering the number of LexA operators upstream of either the LEU2 (EGY191, EGY195) or the lacZ (pJK103, pRB1840) reporter genes. As detailed above, these reporters have been roughly "calibrated" by the systematic comparison of transcriptional activation by interacting proteins with known affinities of association (21), and have been shown to primarily detect interactions occurring at $\mathrm{K}_{\mathrm{d}}$ lower than $10^{-6} \mathrm{M}$. In contrast, some of the additional reagents allow modulation of the expression of the DBD-fusion. For example, pGilda allows inducible expression of LexA-fused baits, making it possible to only transiently express proteins whose continuous overexpression is toxic to yeast. pEE202I is an integrating form of the pEG202 plasmid; use of an integrating rather than a replicating form of this vector results in much lower levels of the DBD-fused protein being expressed, which can be useful for studies of proteins that are normally present at extremely low levels in cells. pJK202 adds nuclear localization sequences to the DBD-fusion, increasing the nuclear concentration of proteins expressed at very low levels or proteins from which it has been impossible to remove all membrane-targeting sequences. These and other vectors possess similar polylinker cloning sites (primarily based on EcoR I-Xho I directional cloning cassettes), facilitating rapid recloning of inserts between different vectors. 
Table 3. List of Interaction Trap strains and plasmids.

\begin{tabular}{|c|c|}
\hline \multicolumn{2}{|c|}{ LexA Fusion Plasmids } \\
\hline pEG202 ${ }^{1^{*}}$ & $\begin{array}{l}A D H \text { promoter expresses } L e x A \text { followed by polylinker: basic plasmid used for } \\
\text { cloning bait. }\end{array}$ \\
\hline pJK202 $2^{2 *}$ & $\begin{array}{l}\text { Like pEG202, but incorporates nuclear localization sequences between LexA and } \\
\text { polylinker: used to enhance translocation of bait to nucleus. }\end{array}$ \\
\hline $\mathrm{pNLexA}^{3}$ & $\begin{array}{l}A D H \text { promoter expresses polylinker followed by } L e x A \text { : for use with baits where } \\
\text { amino-terminal residues must remain unblocked. }\end{array}$ \\
\hline pGilda $^{4}$ & $\begin{array}{l}\text { GAL1 promoter expresses same LexA and polylinker cassette as pEG202: for use } \\
\text { with baits whose continuous presence is toxic to yeast. }\end{array}$ \\
\hline pEE202I $^{5}$ & $\begin{array}{l}\text { An integrating form of pEG } 202 \text { that can be targeted into HIS } 3 \text { following digestion } \\
\text { with Kpn I: for use where physiological screen requires lower levels of bait to be } \\
\text { expressed. }\end{array}$ \\
\hline
\end{tabular}

\section{Activation Domain Fusion Plasmids}

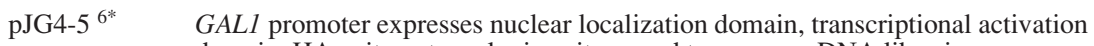
pJG4-5I 5 domain, HA epitope tag, cloning sites: used to express cDNA libraries.

pJG4-5I $\quad$ An integrating form of pJG4-5 that can be targeted into TRP 1 by digestion with Bsu 36I (NEB), to be used with pEE202I to study interactions that occur physiologically at low protein concentrations.

\section{LacZ Reporter Plasmids}

pSH18-34 $7^{7^{*}} \quad 8$ LexA operators direct transcription of the lacZ gene: most sensitive reporter pJK103 $^{2^{*}} \quad 2$ LexA operators direct transcription of the lac $Z$ gene: intermediate reporter for

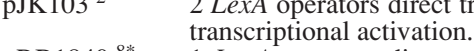

pRB $1840^{8^{*}} \quad 1$ LexA operator directs transcription of the lacZ gene: stringent reporter for

pJK101 2* $\quad$ GAL1 upstream activating sequences followed by 2 LexA operators followed by lacZ gene: used in repression assay to assess bait binding to operator sequences.

\section{Positive and Negative Controls}

pRFHM1 $^{9^{*}} \quad A D H$ promoter expresses LexA fused to the homeodomain of bicoid to produce non-activating fusion. Use as positive control for repression assay, negative control for activation and interaction assays.

pSH17-4 $7^{*} \quad A D H$ promoter expresses LexA fused to GAL4 activation domain. Use as a positive control for transcriptional activation.

\section{LEU2 Selection Strains}

EGY48 ${ }^{1^{*}} \quad$ Basic strain used to select for interacting clones from a cDNA library: 6 LexA EGY191 1 operators direct transcription from the LEU2 gene.

EGY191 ${ }^{1}$ Like EGY48, but with 2 LexA operators rather than 6: a more stringent selection, and producing lower background with baits with intrinsic ability to activate transcription.

Interaction Trap reagents represent the work of many contributors: basic reagents $(*)$ have been described in (8) and were developed in the Brent laboratory, while others have been developed for specialized applications by the individuals noted. ${ }^{1}$ E. Golemis, Fox Chase Cancer Center, Philadelphia; ${ }^{2}$ J. Kamens, BASF, Worcester; ${ }^{3}$ cumulative efforts of I. York, Dana-Farber Cancer Center, Boston and M.Sainz and S.Nottwehr, U. Oregon; ${ }^{4}$ D.A. Shaywitz, MIT Center for Cancer Research, Cambridge; ${ }^{5}$ R. Buckholz, Glaxo, Research Triangle Park; ${ }^{6}$ J. Gyuris, Mitotix, Cambridge; ${ }^{7}$ S. Hanes, Wadsworth Institute, Albany; ${ }^{8}$ R. Brent, Dept. of Molecular Biology, MGH, Boston; ${ }^{9}$ R. Finley, Wayne State University, Detroit. All plasmids and strains can be obtained by contacting the Brent laboratory at Mass. General Hospital (617 7265925 , brent@frodo.mgh.harvard.edu), or they can be purchased from Clontech. 


\section{Protocol}

Control Experiments for DBD Fusions

1. To begin to establish conditions for a two-hybrid screen using the interaction trap, clone the DNA encoding the protein of interest into the vector pEG202. In designing this construction, eliminate any sequences encoding a membrane spanning domain or encoding known transcriptional activation motifs.

2. Co-transform the pEG202-bait construct and the pSH18-34 reporter plasmid into EGY48 yeast. In parallel, transform positive and negative control LexAfusion proteins with pSH18-34 (positive and negative controls for transcriptional activation vary: see reference 20 ).

3. When colonies have appeared, for each transformation, re-streak approximately 10 colonies onto (i) medium containing X-Gal, (ii) onto growth medium lacking leucine, and (iii) onto growth medium containing leucine; glucose should be used as a carbon source in all media. Incubate the plates for 3 days, inspecting on a daily basis.

4. The positive control (for example, LexA-GAL4) should be medium-dark blue on plates containing X-Gal within 1-2 days, and have growth on leucine-minus plates approaching that on leucine-plus plates within 3 days. The negative control should be very pale blue or white on X-Gal plates, and have demonstrated no growth on leucine-minus plates at 3 days. If the positive and negative controls do not behave in this way, something may be wrong with the medium.

The DBD-fusion to the protein of interest being tested may behave in a number of different ways on these media. This behavior will predict the steps to use in preparing it for a screen or other two hybrid study. First, it may demonstrate a lacZ and LEU2 profile similar to the negative control, activating neither reporter. For proteins not known in advance to be transcriptional activators, this is the most likely possibility. If so, the only steps recommended prior to commencing a screen are (i) to perform western blotting analysis of exponentially growing yeast lysates from at least 3 independent colonies to confirm that the protein is being made and that it is the correct size and (ii) to perform a repression assay $(26,20)$ to confirm that the DBDfusion is effectively binding operator sequences. These tests should take approximately 1 week. If the repression assay does not work, the investigator may wish to reclone the bait protein into pJK202, which contains a nuclear localization signal.

A second possible outcome is that the DBD-protein may activate the reporters very strongly, comparably to the positive control. For proteins not known in advance to be transcriptional activators, this seems to occur approximately $5-10 \%$ of the time. For such a protein to be usable in the Interaction Trap or any comparable twohybrid system, it must be truncated to remove the transactivating sequences. Following the truncation, it may be usable in this or another reporter strain/plasmid combination.

A third possibility is that the DBD-protein may activate the reporters moderately - i.e. colonies showing minor growth on leucine-minus medium by the third day after streaking, or becoming light blue on X-Gal medium. These proteins may be usable in a screen with an EGY48/JK103 reporter combination; or they may yield a high background of "false positive" colonies following library transformation. One option is to restart the tests outlined above using a less sensitive set of LEU2/lacZ reporters, such as EGY191 with pJK103 or pRB1840. Alternatively, the protein can be truncated.

Finally, the DBD-protein may grow noticeably less well than the positive or negative control on leucine-plus medium. This outcome may indicate that the DBDprotein is somewhat toxic to the yeast. As this can cause difficulties in performing library screening, it may be desirable to reclone DNA encoding the protein of interest 
into pGilda, which allows inducible expression of the protein from the GAL1 promoter. Note, however, that tests with a pGilda construct should be performed on media containing galactose as a carbon source.

\section{Two-Hybrid Screens}

Depending on the research goals, once a bait with a suitably low transcriptional activation background has been shown to synthesize protein, the next step is either to proceed to screen a library, or to test targeted interactions with other proteins. Library screening usually takes approximately 2 to 3 weeks from start to confirmation of positive clones as specific for a given DBD-protein of interest; these procedures are detailed elsewhere (20). The study of targeted interactions can be accomplished either by individual co-transformation of test pairs, or by an interaction mating approach (27). In this latter approach, panels of LexA-fused proteins are maintained in MATa strains, while additional panels of activation-domain fused proteins are maintained in MAT $\alpha$ strains: cross-plating of the two panels rapidly mates all LexAfused versus all AD-fused combinations, allowing rapid assessment of a large number of interactions. Because of the pseudo-genetic robustness of the two-hybrid approach, simple screening for interaction generally takes a very short period of time. Based on the identity of the interactor isolated, subsequent characterization of the biological significance of the interactor can then take anywhere from weeks to years of effort.

\section{Notes and Tips}

What allows one to gauge whether an interactor is likely to be a real or a spurious partner of the protein of interest? While the final answer will generally depend on extensive functional tests in vivo, there are some general indications.

1. Depending on what is used as DBD-fusion, library screens may yield many, few, or no putative positives. In the cases where many apparent interactors are obtained and where transcriptional background on the part of the DBD-fusion itself has been excluded, it is informative to perform diagnostic restriction digests on a reasonably large number of positives (for example 30-60). If within this number, it is apparent that certain cDNAs are being repeatedly isolated, this suggests that the screen is proceeding with reasonable specificity. Under these conditions, both the frequently and rarely occurring cDNAs may be physiological (with the latter possibly representing interactors occurring at low frequency in the library). Alternatively, analysis of 30-60 positives may suggest that many different cDNAs are being isolated, with no individual cDNA being obtained more than once or twice. If so, this argues that the DBD-fused protein is possibly interacting non-specifically with false positives, and the presumed interactors may be artifactual.

2. Whatever the pattern of cDNA recurrence obtained, plasmids containing putative positives should invariably be retransformed with the original DBD-fusion versus a reasonable number (3-10) of non-related DBD-fusions, to confirm specificity of the interaction with the individual bait.

3. Some proteins appear to be common false positives, being isolated with a large number of proteins. These include HSP70, ribosomal subunits, components of the cellular oxidative phosphorylation pathway, and in some cases, novel proteins of unknown function that sequence analysis suggests might be isolated because of particular sequence traits (such as large hydrophobic patches or long stretches of potential coiled-coil). Formal lists of false positives are only now beginning to be compiled; one source of information can be found on the World Wide Web, at http://www.fccc.edu:80/research/labs/golemis/

4. However, even for proteins which do not fall into the category of common false positives, questions of obvious importance remain. Firstly, it is important to ask if the protein has any conceivable biological relevance to the DBD-fusion used to isolate it. Secondly, one must determine if the protein is known to be 
expressed in an appropriate cell type and intracellular compartment to function in conjunction with the DBD-fusion.

\section{Summary}

In sum, two-hybrid technologies are a very powerful means of applying genetic approaches to the study of protein-protein interactions. Because of the relative ease of working with yeast, these approaches can usually be mastered by any laboratory with basic cloning and molecular biology experience. The majority of proteins tested appear to be amenable to two-hybrid applications. While some are not at this time suitable, efforts are under way in a number of laboratories to address issues such as development of two-hybrid approaches with selection not dependent on nuclear localization or selective activation of transcription. Also, systems are being investigated which would permit more complex screens for interaction between proteins with multiple associating partners, or between proteins and peptides, ligands, or drugs. With the evolution of two-hybrid techniques to allow greater flexibility in the proteins that can be analyzed, and with the ever-increasing diversity of libraries available for screening, the rapid analysis of protein-protein interactions will help to elucidate many signaling pathways of great biological importance.

\section{Acknowledgements}

We are grateful to John Wiley and Sons for permission to use Figures 2, 3, and 4, which also appear in Unit 20.1 of Current Protocols in Molecular Biology, edited by Ausubel, F.M. et al., John Wiley and Sons, New York, 1996. The National Cancer Institute and American Cancer Society provided salary support for the authors.

\section{References}

1. Brent, R. and Ptashne, M. 1985. An eukaryotic transcriptional activator bearing the DNA specificity of a prokaryotic repressor. Cell. 43: 729-736.

2. Johnston, S.A., Salmeron Jr., J.M. and Dincher, S.S. 1987. Interaction of positive and negative regulatory proteins in the galactose regulon of yeast. Cell. 50: $143-146$.

3. Ma, J. and Ptashne, M. 1987. The carboxy-terminal 30 amino acids of GAL4 are recognized by GAL80. Cell. 50: 137-142.

4. Ma, J. and Ptashne, M. 1988. Converting an eukaryotic transcriptional inhibitor into an activator. Cell. 55: 443-446.

5. Fields, S. and Song, O. 1989. A novel genetic system to detect protein-protein interaction. Nature. 340: 245-246.

6. Chien, C.T., Bartel, P.L., Sternglanz, R. and Fields, S. 1991. The two-hybrid system: A method to identify and clone genes for proteins that interact with a protein of interest. Proc. Nat. Acad. Sci. USA. 88: 9578-9582.

7. Durfee, T., Becherer, K., Chen, P.L., Yeh, S.H., Yang, Y., Kilburn, A.E., Lee, W.H. and Elledge, S.J. 1993. The retinoblastoma protein associates with the protein phosphatase type 1 catalytic subunit. Genes Dev. 7: 555-569.

8. Gyuris, J., Golemis, E.A., Chertkov, H. and Brent, R. 1993. Cdi1, a human G1 and $S$ phase protein phosphatase that associates with Cdk2. Cell. 75: 791-803.

9. Vojtek, A.B., Hollenberg, S.M. and Cooper, J.A. 1993. Mammalian Ras interacts directly with the serine/threonine kinase Raf. Cell. 74: 205-214.

10. Vasavada, H.A., Ganguly, S., Germino, F.J., Wang, Z.X. and Weissman, S.M. 1991. A contingent replication assay for the detection of protein-protein interactions in animal cells. Proc. Nat. Acad. Sci. USA. 88: 10686-10690.

11. Fearon, E.R., Finkel, T., Gillison, M.L., Kennedy, S.P., Casella, J.F., Tomaselli, G.F., Morrow, J.S. and Dang, C.V. 1992. Karyoplasmic interaction selection 
strategy: A general strategy to detect protein-protein interactions in mammalian cells. Proc. Nat. Acad. Sci. USA. 89: 7958-7962.

12. Brent, R. and Ptashne, M. 1980. The LexA gene product represses its own promoter. Proc. Nat. Acad. Sci. USA. 77: 1932-1936.

13. Laughon, A. and Gesteland, R.F. 1984. Primary structure of the Saccharomyces cerevisiae GAL4 gene. Proc. Nat. Acad. Sci. USA. 79: 68276831.

14. Munder, T. and Furst, P. 1992. The Saccharomyces cerevisiae CDC25 gene product binds specifically to catalytically inactive ras proteins in vivo. Mol. Cell. Biol. 12: 2091-2099.

15. Fagan, R., Flint, K.J. and Jones, N. 1994. Phosphorylation of E2F-1 modulates its interaction with the Retinoblastoma gene product and the adenoviral E4 19kDa protein. Cell. 78: 799-811.

16. Ruden, D.M., Ma, J., Li, Y., Wood, K. and Ptashne, M. 1991. Generating yeast transcriptional activators containg no yeast protein sequences. Nature. 350: 250252.

17. Triezenberg, S.J., LaMarco, K.L. and McKnight, S.L. 1988. Evidence of DNA:protein interactions that mediate HSV1-immediate early gene activation by VP16. Genes Dev. 2: 730-742.

18. Guarente, L. 1983. Yeast promoters and lacZ fusions designed to study expression of cloned genes in yeast. Methods Enz. 101: 181-191.

19. Bartel, P., Chien, C.T., Sternglanz, R. and Fields, S. 1993. Elimination of false positives that arise in using the two-hybrid screen. Biotechniques. 14: 920-924.

12. Golemis, E.A., Gyuris, J. and Brent, R. 1996. Interaction trap/two-hybrid system to identify interacting proteins. In: Current Protocols in Molecular Biology. F. M. Ausubel, R. Brent, R. Kingston, D. Moore, J. Seidman, S. J. and K. Struhl, eds. John Wiley and Sons, New York. p 20.1.1-20.1.28.

21. Estojak, J., Brent, R. and Golemis, E.A. 1995. Correlation of two-hybrid affinity data with in vitro measurements. Mol. Cell. Biol. 15: 5820-5829.

22. Golemis, E.A. and Brent, R. 1992. Fused protein domains inhibit DNA binding by LexA. Mol. Cell. Biol. 12: 3006-3014.

23. Osborne, M. A., Dalton, S. and Kochan, J.P. 1995. The yeast tribrid systemgenetic detection of trans-phosphorylated ITAM-SH2 interactions. Bio/ Technology. 13: 1474-1478.

24. Ozenberger, B.A. and Young, K.H. 1995. Functional interaction of ligands and receptors of the hematopoietic superfamily in yeast. Mol. Endocrin. 9: 13211329.

25. Sato, T., Hanada, M., Bodrug, S., Irie, S., Iwama, N., Boise, L., Thompson, C., Golemis, E., Fong, L., Wang, H.-G. and Reed, J.C. 1994. Investigations of Bcl2 protein family interactions using the yeast two-hybrid system. Proc. Nat. Acad. Sci. USA. 91: 9238-9242.

26. Brent, R. and Ptashne, M. 1984. A bacterial repressor protein or a yeast transcriptional terminator can block upstream activation of a yeast gene. Nature. 312: 612-615. 
46 Golemis et al. 


\section{Further Reading}

Caister Academic Press is a leading academic publisher of advanced texts in microbiology, molecular biology and medical research. Full details of all our publications at caister.com

- MALDI-TOF Mass Spectrometry in Microbiology Edited by: M Kostrzewa, S Schubert (2016) www.caister.com/malditof

- Aspergillus and Penicillium in the Post-genomic Era Edited by: RP Vries, IB Gelber, MR Andersen (2016) www.caister.com/aspergillus2

- The Bacteriocins: Current Knowledge and Future Prospects Edited by: RL Dorit, SM Roy, MA Riley (2016)

www.caister.com/bacteriocins

- Omics in Plant Disease Resistance Edited by: V Bhadauria (2016) www.caister.com/opd

- Acidophiles: Life in Extremely Acidic Environments Edited by: R Quatrini, DB Johnson (2016) www.caister.com/acidophiles

- Climate Change and Microbial Ecology: Current Research and Future Trend

Edited by: J Marxsen (2016)

www.caister.com/climate

- Biofilms in Bioremediation: Current Research and Emerging Technologies

Edited by: G Lear (2016)

www.caister.com/biorem

- Microalgae: Current Research and Applications Edited by: MN Tsaloglou (2016) www.caister.com/microalgae

- Gas Plasma Sterilization in Microbiology: Theory, Applications, Pitfalls and New Perspectives Edited by: H Shintani, A Sakudo (2016) www.caister.com/gasplasma

- Virus Evolution: Current Research and Future Directions Edited by: SC Weaver, M Denison, M Roossinck, et al. (2016) www.caister.com/virusevol

- Arboviruses: Molecular Biology, Evolution and Control Edited by: N Vasilakis, DJ Gubler (2016) www.caister.com/arbo

- Shigella: Molecular and Cellular Biology Edited by: WD Picking, WL Picking (2016) www.caister.com/shigella

-Aquatic Biofilms: Ecology, Water Quality and Wastewater Treatment

Edited by: AM Romaní, H Guasch, MD Balaguer (2016)

www.caister.com/aquaticbiofilms

- Alphaviruses: Current Biology

Edited by: S Mahalingam, L Herrero, B Herring (2016)

www.caister.com/alpha

- Thermophilic Microorganisms

Edited by: F Li (2015)

www.caister.com/thermophile
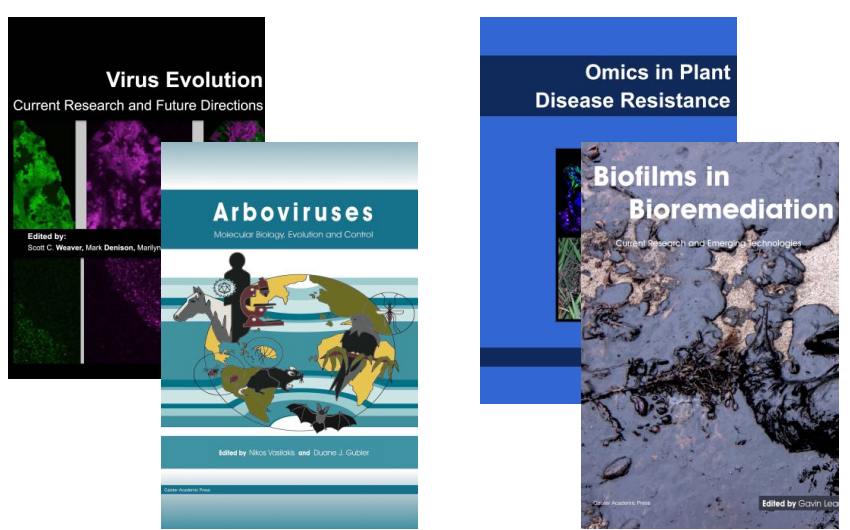
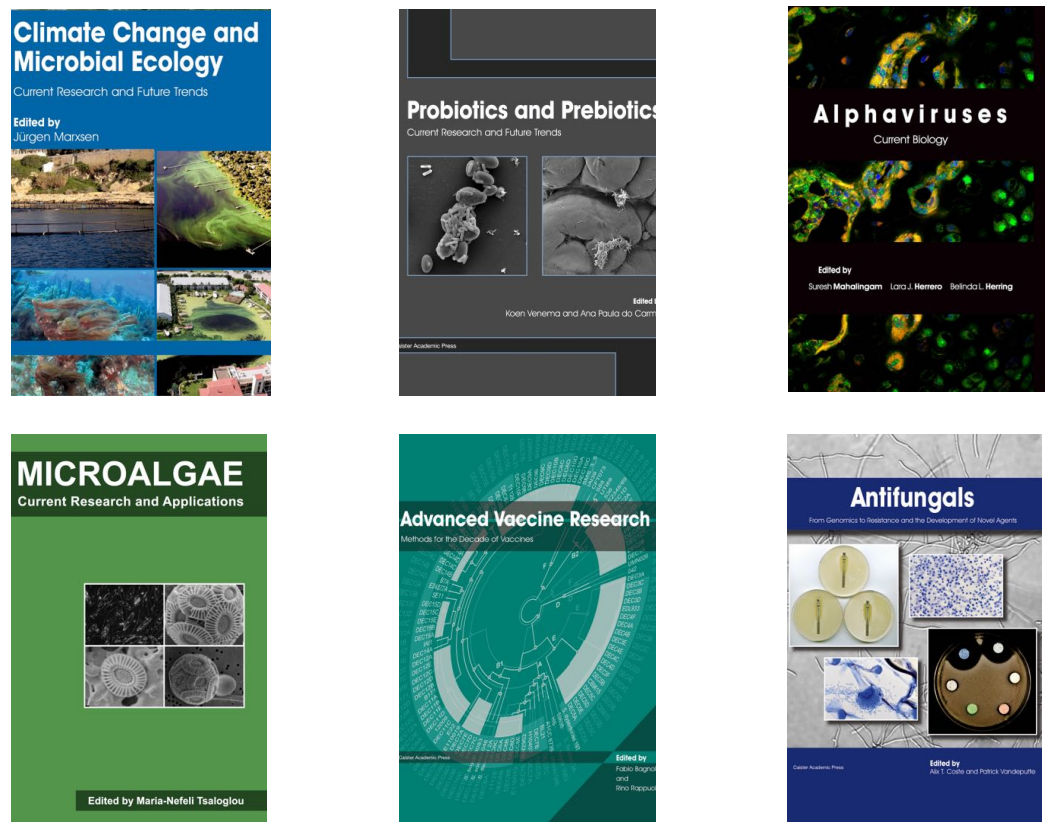

- Flow Cytometry in Microbiology: Technology and Applications Edited by: MG Wilkinson (2015) www.caister.com/flow

- Probiotics and Prebiotics: Current Research and Future Trends Edited by: K Venema, AP Carmo (2015) www.caister.com/probiotics

- Epigenetics: Current Research and Emerging Trends Edited by: BP Chadwick (2015) www.caister.com/epigenetics2015

- Corynebacterium glutamicum: From Systems Biology to Biotechnological Applications

Edited by: A Burkovski (2015)

www.caister.com/cory2

- Advanced Vaccine Research Methods for the Decade of Vaccines

Edited by: F Bagnoli, R Rappuoli (2015)

www.caister.com/vaccines

- Antifungals: From Genomics to Resistance and the Development of Novel Agents

Edited by: AT Coste, P Vandeputte (2015)

www.caister.com/antifungals

- Bacteria-Plant Interactions: Advanced Research and Future Trends Edited by: J Murillo, BA Vinatzer, RW Jackson, et al. (2015) www.caister.com/bacteria-plant

\section{- Aeromonas}

Edited by: J Graf (2015)

www.caister.com/aeromonas

- Antibiotics: Current Innovations and Future Trends

Edited by: S Sánchez, AL Demain (2015)

www.caister.com/antibiotics

- Leishmania: Current Biology and Contro Edited by: S Adak, R Datta (2015) www.caister.com/leish2

- Acanthamoeba: Biology and Pathogenesis (2nd edition) Author: NA Khan (2015)

www.caister.com/acanthamoeba2

- Microarrays: Current Technology, Innovations and Applications Edited by: Z He (2014)

www.caister.com/microarrays2

- Metagenomics of the Microbial Nitrogen Cycle: Theory, Methods and Applications

Edited by: D Marco (2014)

www.caister.com/n2 